
approches théoriques et méthodologiques

\title{
L'atelier culturel en hôpital psychiatrique : un " cadre modalisé », objet de détournements par le personnel soignant
}

Cultural Projects and Psychiatric Hospitals: Uses and Redirections by the Hospital Community

\section{Mylène Costes}

\section{OpenEdition}

\section{Journals}

Édition électronique

URL : http://journals.openedition.org/edc/3888

DOl : 10.4000/edc.3888

ISSN : 2101-0366

\section{Éditeur}

Université Lille-3

\section{Édition imprimée}

Date de publication : 1 décembre 2012

Pagination : 201-216

ISBN : 978-2-917562-08-6

ISSN : 1270-6841

\section{Référence électronique}

Mylène Costes, «L'atelier culturel en hôpital psychiatrique : un «cadre modalisé », objet de détournements par le personnel soignant », Études de communication [En ligne], 39 | 2012, mis en ligne le 01 décembre 2014, consulté le 30 avril 2019. URL : http://journals.openedition.org/edc/3888 ; DOI : 10.4000/edc.3888

Ce document a été généré automatiquement le 30 avril 2019.

(c) Tous droits réservés 


\title{
L'atelier culturel en hôpital psychiatrique : un « cadre modalisé », objet de détournements par le personnel soignant
}

\author{
Cultural Projects and Psychiatric Hospitals: Uses and Redirections by the \\ Hospital Community
}

Mylène Costes

1 Notre recherche se propose d'interroger la mobilisation faite par le personnel des hôpitaux psychiatriques du dispositif de l'atelier culturel, au sein du programme «Culture à l'hôpital ». Résultant d'un accord entre les ministères de la Culture et de la Communication et le ministère de la Santé (1999) ce programme s'inscrit dans la lignée de l'action culturelle en direction des publics dits "éloignés» de la culture. La prise en compte de ces publics par les différents ministères relève d'une volonté de réduction de la «fracture sociale ». A l'hôpital, ce programme, en lien direct avec l'éternel objectif de démocratisation culturelle, vise à favoriser le développement de partenariats entre structures culturelles et établissements hospitaliers.

2 Délaissant la question des enjeux de ce programme pour le public ciblé (personnes en hospitalisation) dont les résultats se recoupent souvent avec ceux d'autres catégories (ex personnes incarcérées, personnes vivant dans les quartiers sensibles), le sujet est abordé sous un angle encore peu développé, celui du personnel hospitalier soignant ${ }^{1}$.

La problématique soulevée concerne l'appropriation faite de l'atelier par les acteurs hospitaliers impliqués ainsi que les répercussions observables auprès de ces derniers. Les études préalablement menées en direction des publics dits «éloignés » laissent entrevoir des «effets » auprès des différents groupes participants à ces ateliers (nouvel espace d'expression, modification des représentations sociales...) (Fabiani, 1995 ; Colin, 1998; Siganos, 2007). Les résultats observés relèvent davantage d'un processus de socialisation que de démocratisation. Nous formulons l'hypothèse qu'à l'hôpital psychiatrique, l'atelier 
fait l'objet d'une réappropriation, voire même d'une forme de détournement (De Certeau, 1990) conduisant à une modification des relations au sein de l'institution hospitalière. Rappelons que dans toute organisation les acteurs, face aux contraintes du système qui leur sont imposées, restent néanmoins en possession d'une certaine liberté qu'ils peuvent utiliser dans une visée stratégique au cours de leurs interactions (Crozier, Friedberg, 1977). Dans l'organisation étudiée, l'hôpital psychiatrique, nous postulons que l'atelier culturel permet l'expression de cette liberté pour le personnel participant notamment car les rapports de pouvoir qui s'y jouent sont autres.

4 Par ailleurs, sachant que des détournements peuvent avoir lieu à l'intérieur d'un cadre (Goffman, 1974a), la problématique de départ sera également traitée en interrogeant en quoi l'atelier culturel peut représenter un nouveau cadre d'expérience, pour le personnel, dans l'enceinte hospitalière. Le dispositif analysé opère une transformation qu'Erving Goffman qualifie de «modalisation $»^{2}$. Nous nous attacherons à comprendre comment des modalisations, en l'occurrence les ateliers culturels, permettent d'interroger les stratégies à l'œuvre chez le personnel et de repenser leurs pratiques professionnelles. Quels changements de "faces $\|^{3}$ ce nouveau cadre qu'est l'atelier dispense t-il ?

5 Si le rôle des membres du personnel est modifié le temps de l'atelier culturel, il paraît intéressant d'interroger la nature de ces modifications et leur tenue dans la durée. Dès lors, sachant que les interprétations que les individus se font des situations qu'ils vivent ont une incidence sur le comportement qu'ils adoptent par la suite (Moscovici, 1989), nous sommes en mesure de penser que des représentations sociales sont produites à la suite des interactions favorisées par l'atelier culturel.

6 Le corpus ici mobilisé, dont sont issus les résultats présentés, est l'objet d'une enquête de doctorat menée auprès de deux établissements hospitaliers psychiatriques : la Fondation Bon Sauveur d'Alby et l'hôpital de Montfavet entre septembre 2008 et janvier 2009. Elle se base sur l'analyse de documents institutionnels (projets d'établissements, projets de service) ainsi que sur une trentaine d'entretiens semi-directifs menés auprès de salariés et artistes intervenants sur les deux sites.

7 Pour répondre au questionnement de départ l'article s'organise autour de trois axes. Le premier vise à définir le dispositif de l'atelier culturel et ses caractéristiques en comparaison aux autres ateliers jusqu'alors menés (art-thérapie). S'ensuit une approche visant à décrire la mobilisation faite de l'atelier culturel par le personnel hospitalier, ainsi que les diverses tactiques qu'il déploie en vue de se l'approprier. Un dernier axe pose la question de l'atelier culturel comme nouveau cadre d'expérience pour le personn el hospitalier, en identifiant les modifications à l'œuvre et notamment les changements de «faces ».

\section{L'atelier culturel : un dispositif majeur au sein du programme « Culture à l'hôpital »}

\section{Place de l'atelier dans le projet culturel}

8 Les applications régionales (conventions entre DRAC et ARS) ${ }^{4} \mathrm{du}$ programme national «Culture à l'hôpital» se concrétisent par la mise en place de projets culturels entre établissements hospitaliers et structures culturelles. Nous n'aborderons pas la dimension partenariale ni même la logique institutionnelle à l'œuvre dans la dynamique du projet, 
nous nous contenterons d'en rappeler les éléments de base pour comprendre la place qu'y tient le dispositif ici analysé à savoir l'atelier.

Tout projet culturel peut être formalisé à travers divers dispositifs : expositions, visites d'établissements culturels, participation à des évènements nationaux. Les travaux de Gilles Herreros, en sociologie des organisations, ne cessent d'en favoriser la compréhension. Retenons notamment que depuis la convention, la création du projet est davantage structurée, planifiée, lui conférant une assise organisationnelle au sein de la structure. Le projet culturel s'est progressivement institutionnalisé, engageant une réflexion commune au sein de l'hôpital (Herreros, Milly, 2009). Il est de plus en plus intégré au projet d'établissement ${ }^{5}$.

Nous envisageons le projet culturel comme objet instituant (Herreros, 2001, 114), il modifie les traditionnels rapports de pouvoir établis par le cadre hospitalier. Il génère des «bouleversements » qui prennent forme essentiellement au sein de l'atelier, permettant la participation commune de patients et du personnel.

11 Pourtant, l'atelier culturel n'a pas encore fait l'objet d'analyse spécifique. Nous pourrions nous demander pourquoi ce dispositif est davantage mobilisé que les autres? On sait grâce aux travaux de Goffman (1974a) que le cadre permet de donner du sens à une action. Les individus se basent souvent sur ce qu'ils connaissent pour développer ensuite leurs propres pratiques. Ainsi nous posons l'hypothèse que l'atelier culturel serait davantage mobilisé car il rappelle les pratiques soignantes effectuées en art thérapie. Pourtant il s'en distingue sur divers points.

\section{Atelier culturel et atelier thérapeutique : éléments de distinction}

Dans le cadre du programme "Culture à l'hôpital», la convention nationale stipule l'obligation d'un atelier de pratique artistique à l'attention des personnes hospitalisées, avec intervention d'un professionnel reconnu. La présence de l'artiste est indispensable, tout comme son absence d'appartenance aux différentes parties en présence. Son extériorité au milieu favorise une libre expression des participants et entraîne auprès d'eux certains changements de "face». Or, en art thérapie il existe peu d'intervenants extérieurs, dans la majorité des cas c'est un infirmier diplômé en art thérapie qui assure l'atelier. Le comportement et les productions des patients y sont systématiquement soumis à analyse car leur participation est intégrée dans un protocole de soins.

De plus, avant la convention, le public visé par ces ateliers était très limité, se composant quasi exclusivement de patients d'un même service. Aujourd'hui, après l'analyse de la convention nationale "Culture à l'hôpital » et le suivi de divers projets, nous pouvons attester qu'il n'existe pas un mais des publics potentiels et que leur implication dans le projet culturel (et donc des ateliers) est de plus en plus manifeste ; ils en deviennent des constituants à part entière. Le public actuel se compose de diverses catégories, essentiellement des patients et du personnel (dont une majorité de soignants), qui grâce à ce nouveau dispositif peuvent multiplier et diversifier la nature de leurs interactions. Le dispositif évolue donc d'une échelle micro (l'unité de soins) à une échelle macro (l'établissement). Il devient accessible à la fois aux personnes en traitement dans les services ouverts et fermés ainsi qu'à ceux hospitalisés en extra (dans les hôpitaux de jour). 
Enfin, l'aspect budgétaire est un autre élément de distinction. En art thérapie, les ateliers sont mis en place sur des budgets internes à l'établissement alors que les financements accordés par la DRAC et l'ARS permettent à l'hôpital de développer d'autres a teliers cette fois d'ordre culturel. Dès lors on peut s'interroger sur leurs rapports avec les ateliers thérapeutiques: y a t-il complémentarité ou mise en concurrence? Pour éclairer ce questionnement il est nécessaire d'analyser la manière dont l'atelier culturel est mobilisé par le personnel.

\section{L'atelier culturel « détourné » dans une logique soignante}

La réflexion suivante s'appuie explicitement sur les travaux de Michel De Certeau (1990). Il s'agit de comprendre ce que le participant «fabrique » au cours de l'atelier culturel. Notre objectif est ici de rendre compte de la production secondaire, autrement dit de la manière et du sens par lesquels le personnel impliqué mobilise ce dispositif.

\section{La participation à l'atelier comme prescription}

16 En effet, dans de nombreux cas, bien que cela n'entre pas dans les clauses du dispositif "Culture à l'hôpital», il est fait appel au médecin de service pour obtenir une prescription de participation à l'attention de certains patients, jugés comme étant des "cas extrêmes", ce qui va à l'encontre de la convention nationale qui prône une participation libre. Ainsi, lorsque le personnel (essentiellement infirmier) estime que la participation d'un patient à l'atelier culturel pourrait avoir un apport bénéfique, il en fait la demande au médecin. Il existe donc une présélection dans l'information communiquée aux patients, tous ne sont pas incités à participer. On peut donc dire que dans les faits l'atelier culturel fait l'objet d'une prescription, intégrant ainsi le cadre de soins proposé au patient. Plusieurs paramètres entrent dans le choix des participants à mobiliser : la pathologie, son stade, ainsi que la durée d'hospitalisation. Les services fermés ont particulièrement tendance à ne proposer l'atelier culturel qu'aux patients hospitalisés en longue durée. Ce phénomène limite l'étendue de l'action culturelle et témoigne de la définition d'un public cible par le personnel alors que le programme "Culture à l'hôpital » veut, dans la mesure du possible, s'adresser à l'ensemble des personnes hospitalisées sans distinction ${ }^{6}$. Indirectement, on pourrait qualifier ces prescriptions comme une nouvelle forme de stigmatisation (bien que non pensé à priori) à l'égard des patients d'hôpitaux psychiatriques. Pour aller plus loin dans la réflexion sur cette attitude, on peut envisager qu'il s'agit pour le personnel de «limiter» les risques en évaluant eux-mêmes préalablement à qui ce dispositif est censé le mieux profiter.

\section{Un nouvel élément pour la prise en charge soignante}

La création est envisagée par les soignants participants comme un indicateur de l'état de santé du patient. La majorité voit dans la pratique artistique une modalité possible du soin. Propice à l'expression, elle permettrait même de l'avis de quelques-uns de limiter les surcharges médicamenteuses, nouvelle trace d'un détournement de l'atelier et plus globalement du projet culturel au profit du soin. 

Herreros, à savoir « le glissement possible de l'exercice du soin à prendre soin » (2008) par le biais cette fois de l'atelier culturel. Au regard du personnel, le projet est exploitable aussi bien dans sa dimension culturelle que dans sa possible dimension soignante. Il est mobilisé dans le soin essentiellement à travers ses caractéristiques socialisantes. Si tous les soignants ne partagent pas l'idée que l'art en soi est quelque chose de thérapeutique, ils conviennent tous que l'atelier culturel est un outil supplémentaire possible pour entrer en communication avec les patients. Au même titre que toute autre activité menée dans le service (jeux, sport, sortie...) il est exploité par le personnel dans la prise en charge quotidienne des patients. La norme que nous avons pu dégager de cette recherche tend à démontrer que l'action culturelle est mobilisée en tant qu'élément à part entière du dispositif de soin. L'équipe soignante ne s'intér esse pas particulièrement à l'atelier, ni au point de vue que peut en avoir le patient. Elle s'intéresse à ce qu'il révèle des potentialités expressives et émotionnelles des patients. Le dispositif est envisagé comme complément au travail effectué par le personnel. Pensé comme élément subsidiaire aux ateliers socio-thérapeutiques, on voit bien que la dimension culturelle de l'atelier n'est pas ce qui retient l'attention du personnel, c'est davantage l'exploitation professionnelle qui peut en être faite.

19 En effet, dans la continuité de ce que nous évoquions quant à la représentation de l'atelier comme outil soignant, nous avons rencontré des services, plus précisément des hôpitaux de jour qui reconnaissent utiliser le dispositif du programme "Culture à l'hôpital » pour développer leurs propres projets, d'ordre thérapeutique ou sociothérapeutique. Le cadre proposé par la convention nationale est avantageux car il représente une autre possibilité d'obtention de financement pour les projets. On peut en déduire que l'atelier culturel (programme «Culture à l'hôpital») fait concurrence aux autres ateliers déjà présents (art-thérapie). Ainsi, pour pallier les restrictions budgétaires et continuer à mener des activités socio-thérapeutiques les infirmiers n'hésitent pas à détourner la convention «Culture à l'hôpital » pour maintenir leurs propres activités, ce qui participe à renforcer le trouble sur la frontière existante entre culture et art thérapie.

\section{Une réexploitation a posteriori}

Arrivé à la fin du projet culturel, les ateliers étant terminés, on assiste à une réexploitation par les soignants des situations vécues. On peut parler d'un détournement à posteriori proche des réflexions menées par André Berten: «Le dispositif échappant maintes fois à celui qui l'a fait naître, est alors détourné, utilisé pour des usages imprévus, remanié pour servir à résoudre tel ou tel problème » (Berten, 1999, 35). En effet, il arrive que le personnel reprenne en actes, dans le service, une partie des situations vécues en atelier. C'est précisément le cas pour les ateliers de pratique théâtrale. Les soignants utilisent le jeu de rôles, notamment au cours des entretiens infirmiers, ils intervertissent les places pour parvenir à débloquer des situations communicationnelles difficiles. Les expériences menées dans le cadre de l'atelier sont vecteurs de dynamisme dans le service. Lors d'un atelier théâtre qui s'est tenu à l'hôpital de Montfavet, une séance a été organisée autour d'un travail sur les percussions corporelles. Durant les jours suivants, ces percussions ont été réutilisées pour assurer le réveil des patients dans le service. Le mouvement s'est étendu à l'ensemble de l'unité s'ad ressant par là même aux patients

Études de communication, 39 | 2012 
n'ayant pas assisté à l'atelier. Ainsi, ce dispositif permet de revisiter le quotidien hospitalier, favorisant la rupture avec une certaine forme de routine.

Ces divers détournements confortent l'idée selon laquelle l'atelier culturel peut représenter un nouveau cadre d'expérience pour le personnel hospitalier en dépassant notamment la simple utilisation professionnelle.

\section{Un nouveau cadre d'expérience dans l'enceinte hospitalière?}

L'interrogation que nous posons ici porte sur la nature des modifications engendrées par la participation du personnel hospitalier à un atelier culturel, en compagnie de personnes hospitalisées. Cette spécificité de l'atelier culturel nourrit l'hypothèse selon laquelle cette expérience participe d'un changement dans les relations soignants-soignés, ce qui nous conduit à interroger les formes effectives et la pérennité de ces changements.

\section{L'atelier culturel comme cadre social}

23 Selon l'approche goffmanienne (1974a), il existe deux grandes catégories de cadres: les cadres naturels et les cadres sociaux. Les cadres naturels appartiennent à ce qui relève de l'ordre du monde physique, alors que les cadres sociaux sont posés par une volonté, un objectif défini. C'est dans cette deuxième catégorie que s'insèrent les actions culturelles menées dans les hôpitaux psychiatriques. Chaque cadre social a des règles qui lui sont propres.

Erving Goffman nous explique qu'une transformation du cadre primaire est une modalisation. En ce sens, le dispositif de l'atelier représente une modalisation du cadre primaire de la relation patient-soignant. Dans le cadre primaire, les échanges patientsoignant sont organisés autour du soin et de la prise en charge, une «barrière » est présente entre les deux entités. Lors d'une participation commune à un atelier culturel par exemple, le cadre est transformé et donne lieu à de nouvelles modalités d'interactions et d'échanges qui ne seraient pas permis en dehors de cet espace. Ainsi, la modalisation résulte de la transformation d'un objet qui avait déjà un sens défini dans un cadre primaire. Cette modalisation (cadre secondaire) est déterminée dans l'espace et dans le temps par certains indicateurs appelés « conventions de phasage » (Goffman, 1974a : 246). Pour l'atelier, il s'agit d'un lieu particulier (souvent en dehors du service) et d'horaires fixes rompant le rythme classique d'une journée d'hospitalisation.

Au-delà de la compréhension de ce qui se joue dans la modalisation de ces cadres à travers l'atelier il s'agit, dans cette étude, de définir les modifications qui peuvent se produire ou non lors du retour aux cadres primaires (dans la quotidienneté de l'organisation et de la vie hospitalière). Dans toute activité, il y a une distinction entre l'individu et le rôle qu'il tient: selon le contexte, c'est la personnalité ou la fonction qui peut prédominer. Chez les soignants, dans le cadre de l'hôpital psychiatrique, c'est le rôle (lié à la profession) qui prédomine tandis que dans l'espace de l'atelier il semble que ce soit la personnalité, l'individu en tant que tel. Il apparaît que la participation à l'atelier permet au personnel soignant d'atteindre un équilibre entre fonction et personnalité difficilement atteignable dans le cadre institutionnel classique. 


\section{Une modification des rôles et des « faces » du personnel soignant}

26 L'action culturelle entraîne un changement dans les comportements, dans les interactions, mais ne modifie pas la nature des rôles des différents acteurs en présence. Un soignant reste avant tout soignant, et un patient reste un malade qui doit être pris en charge. Toutefois ce rôle établi, conventionnel, devient secondaire dans l'atelier culturel. Les rôles ne sont plus figés, mais sont démultipliés, le personnel devient aussi spectateur et acteur tout comme le patient. Plutôt que de multiplicité des rôles, Goffman développe l'idée d'une multiplicité des identités (Ogien, 2005) prenant pour exemple les procédés déployés par un chirurgien lors d'une opération pour s'adresser aux autres membres du corps hospitalier. Pour les ateliers menés dans le cadre du programme "Culture à l'hôpital ", l'individu est avant tout acteur, et c'est cette création commune qui selon nous, permet une multiplicité des identités à l'œuvre et par là même une modification de la nature des interactions.

La notion de «rôle distance » que l'on doit aux travaux de Goffman (1961), entendue comme la possibilité qui est laissée à l'acteur de ne pas s'identifier à son rôle est particulièrement appropriée pour appréhender les positions tenues par le personnel au cours d'un atelier. Elle permet de rendre compte du décalage existant entre la conduite attendue et la conduite réelle, traduisant un refus du soi officiel produit par le rôle (Winkin, 2002). L'espace de l'atelier opère un déplacement (symbolique) des rôles chez le personnel hospitalier.

Tout d'abord dans la "présentation de soi » pour reprendre un terme cher à Goffman (1974b), on assiste à une abolition visuelle et « mentale » de la distinction soignant-soigné entretenue dans le service. Les caractéristiques identificatoires telles que la blouse blanche de l'infirmier sont absentes de manière quasi systématique. Le personnel participant aux ateliers culturels le fait dans sa tenue civile, tout comme le patient et l'artiste. Un ajustement s'opère, entraînant un changement partiel ou plus complet de la face adoptée par le soignant. Il participe d'un rééquilibre du rapport de face entre soignants et soignés.

Dans la pratique, les premières participations des soignants à l'atelier culturel sont vécues de prime abord comme quelque chose d'assez déstabilisant. La principale difficulté est celle du choix de la position à adopter. Le soignant doit être «là ", c'est-à-dire s'intégrer au groupe, sans trop être " là » afin de ne pas déranger, de laisser aux patients la place nécessaire pour s'exprimer. Malgré ces questionnements de départ, tous les soignants rencontrés ont évoqué le désir de se positionner comme les patients, c'est-àdire en tant que participant. Pour comprendre ce qui se joue dans l'atelier, il leur est nécessaire d'intégrer son fonctionnement. La position qui consiste à rester dans l'observation parait totalement inappropriée. Elle est évoquée comme un frein à l'expression des patients. Au sein des services, ils se montrent souvent mal à l'aise lorsqu'ils se sentent observés. Dans le quotidien des séances, les soignants sont avant tout participants, mais assurent aussi un certain encadrement. Ils n'en gardent pas moins un œil discret d'observateur, tout d'abord pou $\mathrm{r}$ vérifier le bon déroulement de la séance auprès de tous les patients, mais aussi dans certains cas pour repérer les bénéfices thérapeutiques. Il y a bien dès lors « simultanéité des identités » (Goffman, 1961).

Mais au-delà, l'atelier culturel permet au personnel de mettre « en retenue » son statut de soignant. Cet espace est le lieu possible du dépassement des codes hiérarchiques et 
relationnels institués dans l'hôpital. À l'instar du patient qui y prend une expression plus libre, le personnel rompt avec son image strictement soignante. Ce positionnement n'apparaît pourtant pas comme quelque chose de réfléchi, mais plutôt comme un comportement spontané, en lien direct avec l'expérience liée à l'atelier culturel. On relève chez les soignants une volonté unanime de se mettre «à l'égal » du patient c'est-àdire en tant qu'individu qui, par sa présence, son implication et ses échanges avec le groupe contribue à la création d'une œuvre. Cette volonté peut être interprétée comme une forme d'insatisfaction voire même de dérangement face à l'attitude de "pouvoir " qu'ils alimentent (consciemment ou mécaniquement) au quotidien, vis-à-vis des patients. Ils prennent plaisir à « abolir ", à mettre en suspens la distance avec leurs patients. Cette participation traduit de leur part une volonté de « démystification » de leur rôle, qui rend plus facile le développement des interactions avec les soignés. Le personnel acquiert une certaine " accessibilité » auprès d'eux car il participe à l'atelier sur une échelle commune, celle des acteurs. Ce constat apparait pourtant comme paradoxal; en effet, au cours de l'atelier culturel le personnel met en suspens sa face professionnelle alors qu'intentionnellement et de manière quasi systématique il participe à ces ateliers dans une visée soignante. Ainsi, la participation se révèle constitutive non seulement de l'identité professionnelle mais également, et peut être même davantage, de l'identité personnelle. C'est le développement de l'identité individuelle au sein du groupe (l'atelier) qui permet de modifier, au moins temporairement, la nature des échanges entre personnel et patients.

De plus, la pratique artistique au sein de l'atelier permet au personnel soignant de prendre conscience de son potentiel. Elle participe à l'acquisition ou plus fréquemment au développement d'une certaine assurance, valorisante sur le plan identitaire. La création apparaît pour ces professionnels du soin comme un mouvement possible vers l'extérieur. De par leurs diverses fonctions, ils sont dans un certain sens "contraints", comme le patient, à n'évoluer en permanence que dans l'enceinte de l'hôpital. Le parallèle peut paraître audacieux, mais nous paraît légitime. Bien que ces professions soient souvent pratiquées par choix, voire vocation, cela n'empêche pas la présence (souvent évoquée lors de nos entretiens), chez les professionnels, d'un sentiment d'étouffement et de routine qui semble peser lourd sur leurs pratiques quotidiennes. La création au sein de l'atelier culturel permet donc au personnel d'être dans un extérieur du soi (le soi de soignant), de créer une rupture temporaire avec l'univers hospitalier autour duquel il gravite au quotidien. Les acteurs prennent conscience de la retenue à laquelle les contraint leur profession et combien il peut être difficile de la dépasser.

Une certa ine prise de distance est rendue possible le temps de l'action culturelle. Elle permet au personnel de mettre de côté son rôle professionnel au profit de son rôle de citoyen, favorisant dès lors le développement d'une conscience sociale et non plus strictement professionnelle.

\section{Vers de nouvelles interactions « soignants-soignés »}

À travers le nouveau cadre d'expérience qu'il représente, l'atelier devient une "scène " appropriée au développement d'interactions entre hospitaliers et hospitalisés. Elles sont en partie permises par la disponibilité totale dont fait preuve le personnel lors de ces séances. En effet, aucun facteur extérieur, aucune formalité liée au fonctionnement du service ne peut venir troubler l'implication et la présence du personnel auprès des 
patients. L'atelier culturel apparaît comme le seul espace dans l'établissement hospitalier où les soignants peuvent se rendre totalement présents pour leurs patients.

Les changements de face opérés entraînent une modification des relations entre les acteurs au cours des ateliers. Une certaine proximité, entendue dans son sens relationnel, se développe. Les soignants ne sont plus soumis à l'activité du service, aux contraintes soignantes et horaires, ils passent en mode «disponible » auprès des patients. Ainsi, les acteurs ne sont pas en situation de soins, le rappel des règles de l'organisation hospitalière n'est plus présent explicitement. Dès lors, pensée comme la personnalisation de la société, la face des acteurs prend un autre sens lors de l'interaction dans l'atelier. Cet espace laisse place davantage au développement et à la manifestation de l'individualité vis-à-vis de la société, ce qui explique par ailleurs le «délaissement » du contrôle exercé sur la face par le personnel hospitalier. Il en ressort une prédominance de la personnalité de l'individu sur son rôle. C'est dans l'espace de l'atelier culturel que se formalise la distance au rôle telle que l'a défini Goffman et que de nouvelles interactions peuvent émerger.

\section{Conclusion}

Nous avons mis en lumière la manière dont les individus (en l'occurrence ici le personnel hospitalier) sont capables de détourner des dispositifs, de se les réapproprier, vérifiant ainsi notre hypothèse de départ. L'atelier culturel fait l'objet de détournements par le personnel essentiellement dans une logique soignante, de complément de prise en charge mais également de logiques financières pour maintenir sous une autre forme des dispositifs socio-thérapeutiques. Il y a donc à la fois complémentarité et concurrence entre ces deux catégories d'ateliers.

Au-delà de ce constat, la recherche permet de comprendre l'apport du dispositif pour l'individu soignant. L'atelier lui permet non seulement de revisiter ses pratiques professionnelles mais également d'engager une réflexion d'ordre plus personnel. Cela favorise des changements de «faces » donnant lieu à de nouvelles interactions avec les patients, basées cette fois sur l'échange et non strictement sur le soin. Pourtant, on retiendra une tension importante entre la volonté émanant du personnel de sortir du cadre de soins (cadre primaire de l'hôpital) et l'impossibilité manifeste de le faire au sein de l'atelier culturel.

Ainsi, il serait erroné de penser que ces modifications de faces tout comme la nature de ces interactions puissent perdurer au-delà de l'atelier. En plus d'un retour au cadre primaire et à la rigidité du cadre de l'hôpital psychiatrique, d'autres éléments rendent caduque un tel maintien. Il s'agit bien évidemment de la persistance des rites hospitaliers dans l'organisation (Pouchelle, 2003) mais surtout de ce désir du personnel de ne pas perdre la «face». Le besoin de sauver la face n'est pas un besoin individuel, mais une exigence de la société parlant en nous comme l'a souligné (Goffman, 1974b). Dès lors, l'atelier culturel n'est pas en soi un nouveau cadre au sein de l'hôpital psychiatrique mais davantage un sous-cadre.

Il n'en reste pas moins qu'au sortir c'est dans le regard qu'il porte sur ses patients que s'opère une modification chez le personnel hospitalier. D'autres représentations sociales sont la trace manifeste des " effets » à long terme de l'atelier culturel. Bien que chacun retrouve sa fonction stricte (soignant et patient) il n'en reste pas moins qu'un 
«déplacement » du regard s'est opéré. Notre constat complète celui soulevé par Gilles Herreros : "C'est le regard de l'autre qui bouge » (2008). Ce dispositif produit donc des perturbations, plus ou moins visibles dont la durée s'inscrit au-delà de l'éphémère. Il traduit ainsi la porosité des cadres de l'institution hospitalière.

Enfin, le regard ici posé sur le dispositif de l'atelier réinterroge la prise en charge des personnes hospitalisées. Pour dépasser de manière concrète le débat encore latent entre soins et culture il conviendrait de les penser davantage en complémentarité, ce que devrait permettre la multiplication des ateliers culturels. De plus, nous avons vu que l'atelier en tant qu'accès possible à la culture, démarche citoyenne envers des personnes hospitalisées ne semble pas suffire pour interpeller significativement le personnel hospitalier. C'est selon nous du côté de la prise en charge globale que la dynamique pourrait se voir généraliser. La dernière con vention signée nous conforte dans cette idée. En effet, le 06 mai 2010 le Ministère de la Santé et des Sports et le Ministère de la Culture et de la Communication ont signé une nouvelle convention dont le seul titre est déjà évocateur: "Culture et Santé ». On assiste ici à une reconnaissance de l'enjeu que peut représenter la culture dans l'amélioration de la prise en charge des personnes hospitalisées ainsi que de leur guérison. Pour le ministère de la Culture cela n'a rien d'anodin et traduit un dépassement du traditionnel discours sur l'objectif de démocratisation culturelle. Espérons que les établissements hospitaliers sauront collectivement s'en saisir.

\section{BIBLIOGRAPHIE}

Berten A., (1999), Dispositif, médiation, créativité, petite généalogie, in Hermès, n² 25, pp. 33-45.

Colin B., (1998), Action culturelle dans les quartiers : Enjeux et méthodes, Culture et Proximité hors série.

Crozier M. et Friedberg E., (1992), L'acteur et le système, Paris, Seuil, 500 p. [Publication originale de 1977].

De Certeau M., (1990), L'invention du quotidien, 1. arts de faire, Saint-Amand, Gallimard, 349 p. [Publication originale de 1980].

Fabiani J.-L., Soldini F. et Rigot H., (1995), Lire en prison : une étude sociologique, ADCSHAM, Ministère de la Justice, Direction du livre et de la lecture, La Documentation française.

Goffman E., (1961), Role Distance, in Encounters, Indianapolis, Bobbs Merrill, 152 p.

Goffman E., (1968), Asiles : Études sur la condition sociale des malades mentaux, Paris, éd. Minuit, $447 \mathrm{p}$.

Goffman E., (1974a), Les cadres de l'expérience, Paris, Minuit, 576 p.

Goffman E., (1974b), Les rites d'interaction, Paris, Minuit, 230 p.

Herreros G., (2001), Identités professionnelles et dynamique de changement, in Il suffit de passer le pont : restitution du groupe de réflexion régional « culture à l'hôpital », pp. 99-118, http : // 
www.culture.gouv.fr/rhone-alpes/hopital/image/textpont.pdf, date de la dernière visite : 25 octobre 2011.

Herreros G., (2008), Intervention lors du séminaire « Culture, hôpital et territoires », Lyon, le 13 mai 2008, http://podcast.univ-lyon2.fr/groups/culturehopitaletterritoires/webblog, date de la dernière visite : le 26 mai 2009.

Herreros G. et Milly B., (2009), Culture-Hôpital : De l'expérimentation à l'institutionnalisation, Rapport de recherche, ARH, DRAC, Région Rhône-Alpes, http:// www.culture.gouv.fr/rhonealpes/hopital/pdf/rapport-herreros-2009.pdf, date de la dernière visite : 16 avril 2010.

Ministère de la Culture et de la Communication et Ministère de la Santé, (1999), Convention nationale «Culture à l'hôpital ».

Moscovici S., (1989), Des représentations collectives aux représentations sociales : éléments pour une histoire, in Les représentations sociales (dir. Jodelet), Paris, PUF, pp. 62-86.

Ogien A., (2005), Le remède de Goffman ou comment se débarasser de la notion de self, in Séminaire Cesames, le mental, le vivant, le social, 20 janvier, http://cesames.org/seminaires.htm, date de la dernière visite : 15 octobre 2010 .

Pouchelle M.-C., (2003), L'hôpital corps et âmes, Paris, Seli Arslan, 218 p.

Siganos F., (2007), L'action culturelle dans les prisons françaises : Formes et Enjeux, Thèse de Doctorat de $3^{\mathrm{e}}$ cycle : Sociologie/sous la direction de Jean Marc Leveratto : université de MetzPaul Verlaine.

Winkin Y., (2002), Erving Goffman, in Elliott A. et Ray L., Key Contemporary Social Theorists, Londres, Blackwell, pp. 140-147.

\section{NOTES}

1. Sous le terme "soignant» nous entendons essentiellement les infirmiers et aides-soignants qui regroupent les catégories professionnelles de l'hôpital les plus impliquées dans les ateliers culturels.

2. «Par mode j'entends un ensemble de conventions par lequel une activité donnée, déjà pourvue d'un sens par l'application d'un cadre primaire, se transforme en une autre activité qui prend la première pour modèle mais que les participants considèrent comme sensiblement différent. On peut appeler modalisation ce processus de transcription » (Goffman, 1974b : 54).

3. Erving Goffman considère comme «face»: "la valeur sociale positive qu'une personne revendique effectivement à travers la ligne d'action que les auteurs supposent, qu'elle a adoptée au cours d'un contact particulier $»(1974 b: 9)$.

4. Suite à la loi du 21 juillet 2009 (HPST) portant réforme de l'hôpital et relative aux patients, à la santé et aux territoires, les ARH (agences régionales d'hospitalisation) ont été remplacées par la création d'ARS (agences régionales de santé) dont l'objectif est d'assurer le pilotage de la santé en région.

5. Nous renvoyons le lecteur vers les travaux de Patrick Basse (2003), en sciences de l'information et de la communication, sur le projet d'établissement, qui peuvent aider à penser la place du projet culturel au sein de l'institution hospitalière dont nous ne traiterons pas ici.

6. Le préambule de la convention nationale «Culture à l'hôpital » stipule : « La culture peut jouer un rôle essentiel dans cette évolution. En dehors de tout objectif thérapeutique, elle participe à l'amélioration de l'environnement des personnes et contribue à favoriser la relation de l'hôpital 
avec l'extérieur » (extrait de la convention nationale «Culture à l'hôpital », ministère de la Culture et de la Communication, ministère de la Santé, 1999).

\section{RÉSUMÉS}

Notre travail interroge les usages faits par la communauté hospitalière des projets culturels menés au sein du programme «Culture à l'hôpital». Prenant pour cadre les hôpitaux psychiatriques, l'approche se veut institutionnelle, délaissant le public visé par ce programme (personnes hospitalisées) pour se concentrer sur les hospitaliers. Comment les professionnels investissent-ils ces projets, de quelles manières cela se traduit-il ? Nous défendons l'hypothèse selon laquelle ces projets sont objet de détournements (De Certeau, 1990), de réappropriations dans des logiques diverses, qu'elles soient managériales ou communicationnelles pour les directions ou professionnelles pour le personnel soignant. De même, ces multiples détournements viennent bousculer la ritualisation à l'œuvre dans l'organisation hospitalière interrogeant la place du projet culturel dans la redéfinition des identités professionnelles.

Our work examines the uses made by the hospital community of the projects carried out within the "Culture in the Hospital" program. Taking psychiatric hospitals as a framework, the approach aims to be institutional, forsaking the public targeted by this program (i.e., hospitalized people) in order to concentrate on the hospital setting. How do professionals invest in these projects, and what does this investment translate to? We defend the view that these projects are susceptible to redirection (de Certeau 1990), re-appropriation through various managerial logics that are communication-related for management or professional for medical staff. Similarly, these multiple redirections upset the ritualization at work in the hospital organization, creating a need to question the place of the cultural project in the redefinition of professional identities.

INDEX

Mots-clés : projet culturel, hôpital, usages, détournement, identités professionnelles

Keywords : cultural project, hospital, uses, diversion, professional identities

\section{AUTEUR}

\section{MYLÈNE COSTES}

Mylène Costes - Université Grenoble II - GRESEC 